\title{
Generation and Simulation of Indoor Thermal Gradients: MRT for Asymmetric Radiant Heat Fluxes
}

\author{
Dorit Aviv ${ }^{1,3}$, Eric Teitelbaum ${ }^{1}$, Tyler Kvochick ${ }^{1}$, Kipp Bradford ${ }^{1}$ Forrest Meggers ${ }^{1,2}$ \\ ${ }^{1}$ Princeton University School of Architecture, Princeton, NJ, USA \\ ${ }^{2}$ Andlinger Center for Energy and the Environment, Princeton, NJ, USA \\ ${ }^{3}$ University of Pennsylvania Weitzman School of Design, Philadelphia, PA, USA
}

\begin{abstract}
The inherent geometric and material dependence of radiant heat transfer can be leveraged to improve system efficiency and thermal comfort. Unlike in air-based systems, non-uniform temperature distribution can be highly controlled and beneficial in radiant systems, where temperature perception can be manipulated locally. An experiment was devised with the aim of creating a significant temperature gradient in a single space by using radiant heat transfer to cool certain parts of a room while simultaneously heating other parts. This was achieved by inducing radiant fluxes from hot and cold emissive pipes and directing them at different areas of the room through the use of curved infrared reflective surfaces. A 3D simulation was created to analyze the consequences of such a configuration for the Mean Radiant Temperature (MRT). The simulation utilizes a ray-tracing technique to account for multiple reflection bounces. The results are compared to MRT measurements taken in the physical experiment using Black Globe Thermometers. A simulation study of the heat transfer characteristics of a single pipe in a parabolic trough is also discussed.
\end{abstract}

\section{Introduction}

The human body is a complex thermal engine, with multiple sophisticated mechanisms for exchanging energy with its environment. Air temperature is just one of many factors that have significant physical impacts on perceived comfort, yet thermostats measuring only air temperature control how buildings deliver thermal amenities, ignoring many opportunities to affect human comfort through the design of a room. More comprehensive human comfort models, as discussed in Huizenga et al (2001), recognize a wide set of influences from convection, conduction and radiation between the body and the environment. In the following experiment, the focus on control and manipulation of the radiant heat exchange in an indoor environment, using specific material and geometric properties of the surfaces in a room, contributes to a shift towards thermal comfort modelling based on an operative temperature that decouples air temperature and mean radiant temperature (MRT).

MRT is an important factor in calculating the radiant heat exchange between the human body and the surrounding environment. It is defined as the mean temperature of all the surfaces surrounding a human body in space, weighted by the view factor of each surface in relation to the body. Therefore, using a specific geometric configuration to increase or decrease the view factor of a heat source in relation to the body of a human occupant, it is possible to magnify or reduce the radiant exchange between them.

Simulation techniques of MRT have largely been developed for urban climate studies (Matzarakis et al, 2007), where MRT is calculated based on view factor derived from urban fisheye photography. However, when considering diffuse longwave radiation in an enclosed room with complex geometry and varying emissivities causing significant reflections, the common assumptions in large urban settings of diffuse interactions necessitates a different simulation method to be employed. For such purpose, an indoor simulation tool which enables predicting the effects of solar radiation on human heat gain and comfort Schiavon et al, 2015). However, a high-resolution indoor longwave radiation simulation with multiple radiosity bounces can be found in radiosity simulations developed in the field of computer vision (Cohen and Greenberg, 1985).

Thermal Radiation, being within the infrared spectrum of electromagnetic radiation, shares many of the characteristics of visible light but occupies a different spectrum of wavelengths. As such, it is also possible to manipulate the direction of a heat source using a curved reflective surface around it, similarly to the use of a parabolic extrusion for a light fixture around a cylindrical light bulb. There are examples of patents that used exactly this concept (Heck, 1941), where a device is constructed to concentrate radiant heat or shield objects from heat for use in medical procedures requiring heating and cooling. In this paper, the simulation of the thermal behaviour of a parabolic reflector, directing radiant flux from a hot or cold pipe will be discussed.

Beyond the scale of a single heat reflector, the Thermoheliodome project (Meggers et al, 2017) provides an example of a constructed demonstrator of reflective expansion of radiant heat transfer. The project provided cooling to occupants through the reflection of thermal radiation emitted from chilled pipes using conical concentrators to provide cooling without conditioning the air. For outdoor thermal comfort, the provision of heating or cooling radiatively was 
demonstrated to be more than $80 \%$ more efficient than an air-based system due to the separation of the radiant surface from the area available for radiant cooling, thereby reducing convection proportionally to this reduction. The reflective expansion in an outdoor environment has similar embodiments in the indoor built environment, allowing for thermal gradients to be established without relying on air to provide the thermal energy.

With Thermoheliodome, the architectural scale prototype was successful in demonstrating the implications of radiant cooling resulting from several smaller modules, compared to the contributions from singular radiant panels or reflectors, which is the conventional design approach. However, comfort was directed at a point in space. With the experiment discussed in this paper, the goal shifted instead to providing a thermal gradient, or comfort gradient through a space and allowing users to direct themselves to their physiologically dictated comfortable point.

Variable temperature distribution is considered a problem when an air-based mechanical system is employed in an indoor space. However, nonhomogeneous temperature distribution in a room with a radiant system can be beneficial (Roulet et al, 2005). Radiation-based systems allow for local heating or cooling. By directing heat fluxes towards a specific area of a room rather than trying to condition a large volume of air uniformly, greater efficiency can be achieved.

Radiant cooling panels, especially ceiling panel systems are becoming more prevalent in buildings today. However, their design is usually limited to modular flat ceiling panels and rarely takes advantage of geometric manipulation of heat flux directionality. One such rare example is the vaulted radiant ceiling installed at the Richard J. Klarchek Information Commons at Loyola University (McLauchlan et al, 2008), where non-flat surfaces are used in a full-scale architectural installation to produce radiant heating and cooling effects. However, in this example, the parabolic ceiling is used for indirect lighting rather than for magnifying the radiant heat exchange.

\section{Methods}

The goal of the experiment was to control MRT gradients in a room using strategically surface geometry to expand or minimize the view factor of small heat source surfaces. By using reflective parabolic troughs, it was possible to create discrete thermal programming, where a person standing at different regions of the same space perceives significantly different comfort conditions. This has the potential of raising the efficacy of radiant heating and cooling in buildings: instead of creating uniform environments with volumetric airflow comfort control, manipulating surface radiation makes it possible to cool or heat specifically occupied regions of a room strategically. In order to achieve this, it was necessary to develop MRT simulation techniques that enabled predicting and optimizing the results of the reflective geometry. Thus, the following series of studies were aimed at developing a radiant reflection technique to create a controlled indoor thermal gradient:

- Heat flux from a single pipe and a single parabola

- Room-scale physical prototype

- MRT simulation technique development

\section{Heat Flux from a Single Pipe and a Single Parabola}

A pipe is used as a heat source with a parabolic extrusion that manipulates the effect of the radiant flux from a pipe on a human body standing across from it. The parabolic surface reflects the heat flux from the pipe back towards the person (See Fig. 1). The pipe is placed at the focal point of the parabola and thus the reflected rays emitted normal to the pipe surface become parallel to each other.

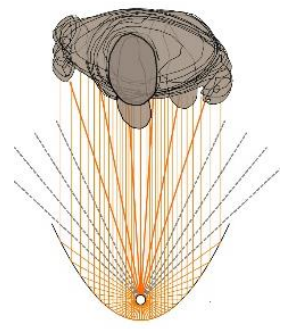

Figure 1: Reflection of heat flux from a parabolic trough with a pipe at the focal point onto a person.

We first characterize the parabolic shape in relation to the pipe size and location: for a physical parabolic extrusion, we chose a $46 \mathrm{~cm}$ width, a rough estimate of the width of a person's torso. If a parabola with a vertex on the origin $(0,0)$ point, is defined by:

$$
y=a x^{2}
$$

as $a$ increases, the parabola is steeper, the focal point is closer to the origin. We look at the consequences for the resulting heat flux. If we assume the reflector surface to be fully specular, then the radiant flux leaving it is equal to the radiant flux from the portion of the surface of the pipe optically encased by the parabolic surface. We simulate the percentage of radiant flux from the pipe reflected back to the person standing in front of it by creating an array of vectors from the normal of the pipe subdivision and comparing the changed parabolic shape to the ratio of reflected rays.

Next, we characterize the radiant heat flux from the pipe in relation to convective losses. To calculate the total heat flux from a single pipe we use

$$
Q_{\text {tot }}=Q_{\text {rad }}+Q_{\text {conv }}
$$

Where $Q_{\text {rad }}$ is the radiative heat flow from the pipe $Q_{\text {conv }}$ is the convective heat loss (or gain) and $Q_{\text {tot }}$ is the total heat transfer from the surface of the pipe.

For a given pipe temperature $T_{p}$ which we can control by regulating the water temperature in the pipe:

$$
Q_{\text {rad }}=\sigma T_{p}^{4} A
$$

where $\sigma$ is the Stefan-Boltzmann constant, $5.67 \times 10^{-8}$ $\left(\mathrm{W} / \mathrm{m}^{2} \mathrm{~K}^{4}\right), T_{p}$ is the surface temperature of the pipe in Kelvin (K), and $A$ is the surface area in $\mathrm{m}^{2}$ of the pipe: 
$2 \pi r l$, where $r$ is the radius of the pipe and 1 is the length in meters.

$$
Q_{c o n v}=h A\left(T_{p}-T_{a i r}\right)
$$

Where $h$ is the convection coefficient in $\mathrm{W} /\left(\mathrm{m}^{2} \mathrm{~K}\right)$, and will be driven largely by free convection from buoyant forces caused by the difference in the pipe and air temperature This creates a complex variation in convective flux, which will also have a secondary influence of any small forced convection due to dynamics of the building openings and systems. Looking at free convection values and small forced convection mixed regimes, the convection coefficient should range from 5 to $10 \mathrm{~W} / \mathrm{m} 2 / \mathrm{K}$ ( $\mathrm{Li}$ and Tarasuk, 1992 and Boetcher, 2014). For the cold and hot pipes in this experiment, we calculated $\mathrm{h}$ values of 6.4 and 7.6 $\mathrm{W} / \mathrm{m}^{2} / \mathrm{K}$, respectively, from the Nusselt number correlations listed in Boetcher (2014). Because of the convective losses, the surface temperature of the pipe will be reduced when hotter than the air and increased when colder. The combination of Qrad and Qconv will result in an equilibrium temperature of the pipe surface delivered by convection from the working fluid inside and the conduction through the pipe material.

If we consider a person standing in front of the parabola with focal pipe, the radiant exchange with the pipe includes the view factor of the surface of the pipe, plus the radiant flux reflected from the parabolic surface (Fig. 1). The use of this method therefore greatly increases radiation's efficacy in comparison with a conventional radiant panel with the same energy input from an identical size water pipe (Fig. 2). This is because for the same effective panel length $d$, a conventional panel will produce losses in conduction over the flat panel and convection losses over the full length $d$ where a parabolic trough will only have convective losses around the much smaller surface of the pipe itself, and small amount of losses due to heat flux from the pipe which is not captured by the parabola (see Fig. 1).

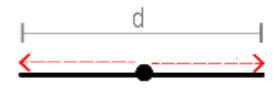

(a)

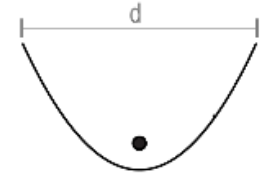

(b)
Figure 2: conventional radiant panel (a) with water pipe heat source conducting to the rest of a flat surface versus a parabolic trough $(b)$ with the same size water pipe, with no conduction and reduced convective losses.
Next let us examine the effect of the position of a person in space in relation to the parabola. Because a person's surfaces emit diffusely the rays simulated from a point emit in all directions, and therefore are not parallel like the rays reflected by the parabola from the pipe discussed above. We examine the ideal location for a person for maximum radiant exchange with the pipe. Because the pipe has a width, it spans across the focal point of the parabola, and it captures incoming rays that are not parallel. We define a "convergence point" based on the distance where the width of the pipe captures all angles of emission that are intercepted by the parabola (see Fig. 3 and 4c). Using similar triangles to show the relationship between the pipe diameter, the focal point, $r$, the focal length of the parabola, $f$, and the convergence distance, $d$, one can arrive at the following relationship for designing convergence shown in equation 4. This method is easily visualized for a latus rectum of the parabola, however it generalizes easily with the use of the sine law and similar triangle properties.

$$
d=r \sqrt{1+\frac{f}{r}} \sin \left(\frac{\pi}{2}-\tan ^{-1}\left(\frac{f}{r}\right)\right)
$$

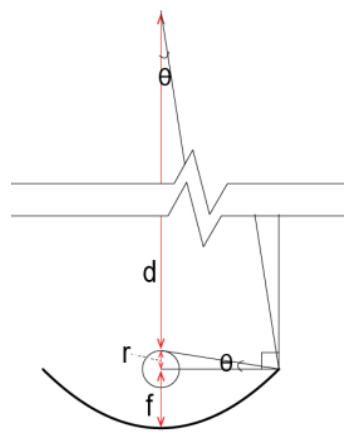

Figure 3: Illustration of Equation 5 parameters defining the distance to the point from where the width of the pipe captures all angles of emission that are intercepted by the parabola.

If we look from the perspective of a point in space, the convergence point of the parabola becomes significant and moving from it either sideways or closer to the parabola reduces the radiation reflected from the pipe and hitting that point (see Fig. 4). The pipe size and parabolic shape were held constant. The result section will detail the rate at which the reflected heat flux changes based on the front and sideways distances from the point of convergence.

We have also built a physical prototype of a single parabola and a pipe to verify the simulation results compared with infrared imaging of the physical prototype. 


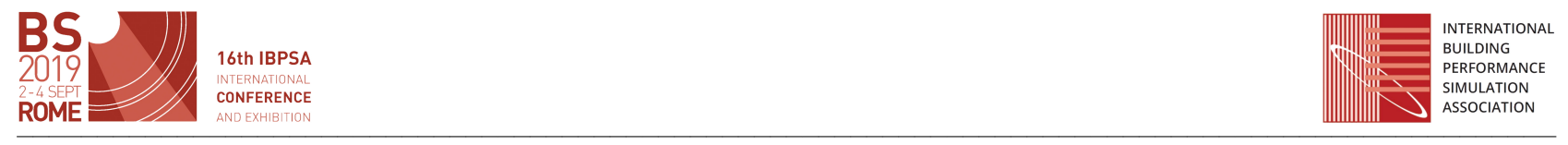

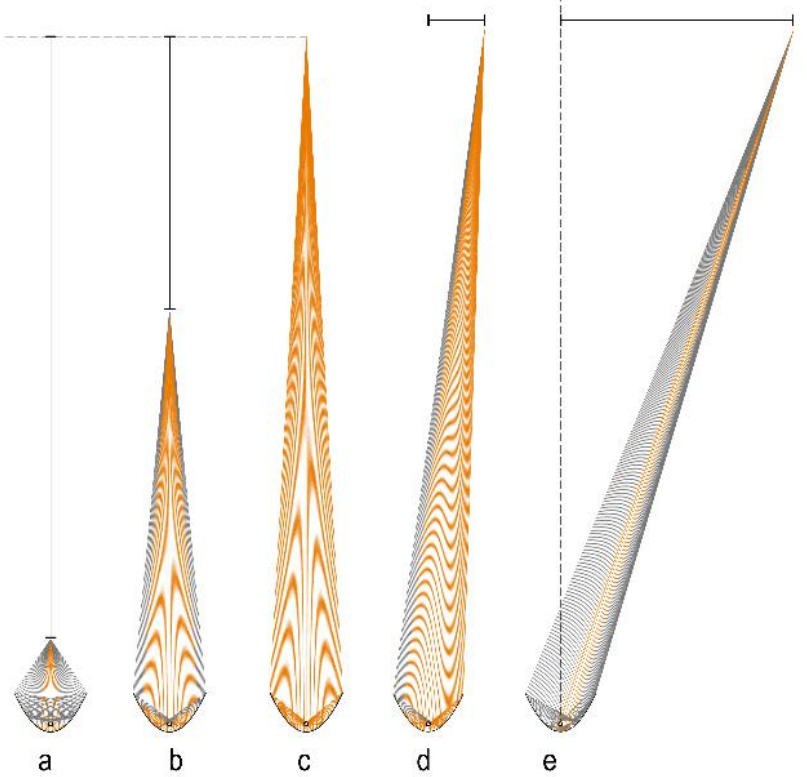

Figure 4: diagram illustrating the convergence point (c) and deviations to the front $(a, b)$ and sideways $(d, e)$.

\section{Room-Scale Physical Prototype}

In order to validate the simulation technique, which we will discuss in the next section, we constructed a roomsize physical experiment with interior dimensions of $4 \mathrm{~m} \times 2 \mathrm{~m} \times 2.4 \mathrm{~m}$ and walls and ceiling made of curved aluminium surfaces around closed loops of emissive hot and cold water pipes (Fig. 5 and 6). The water temperature in the pipes was regulated by a highefficiency heat pump (full description of the thermodynamic cycle and pump characterization to be discussed in a separate paper).

We used the following measurement tools to evaluate radiant and convective effects:

1. Black Globe Thermometers were placed at three points in the room: front end, rear end and the middle point of the room. They were all placed mid-height at $1.2 \mathrm{~m}$ from both floor and ceiling.

2. DHT22 sensors were used to determine the airtemperature at the black globe locations.

3. Infrared photography was used to determine the surface temperature of the pipels.

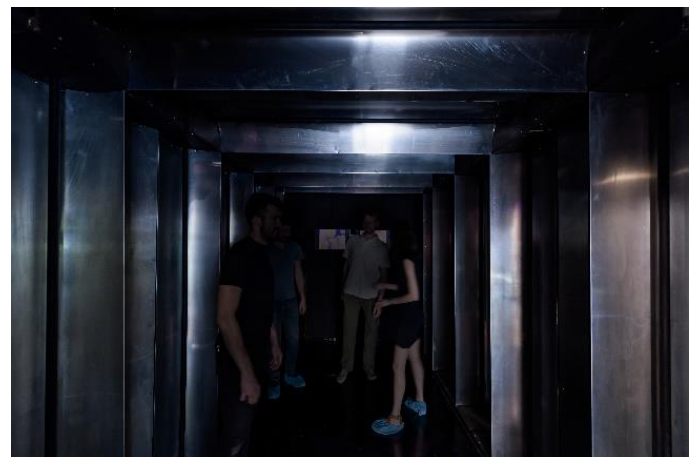

Figure 55: view of physical experiment as built, with people inside the room.

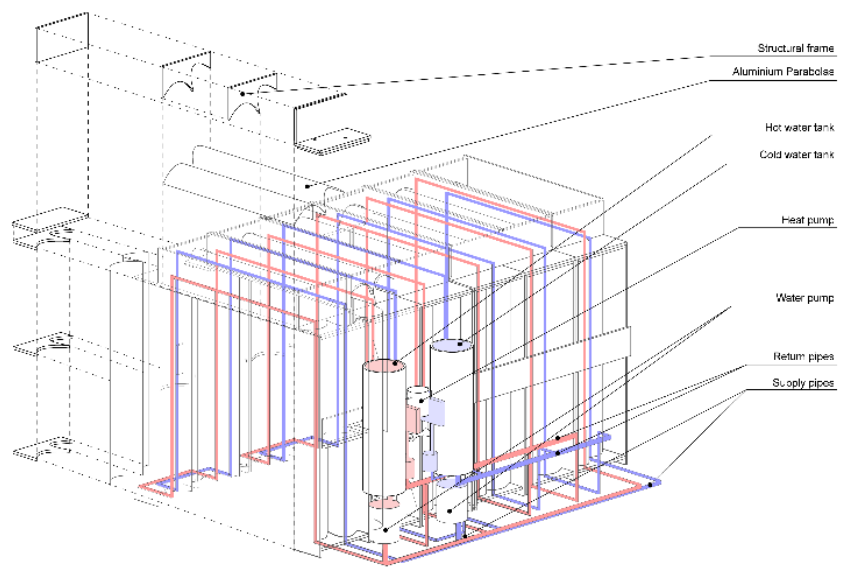

Figure 6: 3D diagram of water pipe circulation.

The results measured in the physical experiment were used as the basis for surface temperature for the 3D simulation described in the next section.

\section{MRT Simulation Development}

As mentioned in the introduction, there are several existing MRT simulation techniques, but many of them were developed for assessment of urban outdoor comfort rather than for indoor environments, while some ignore reflections and others use generalized geometry. In order to simulate the conditions we have created in our experiment accurately, which are governed by multiple heat sources and a highly specific geometric configuration which results in multiple reflections, we developed a 3D ray-tracing simulation in Grasshopper algorithmic modeling tool, which accounts for accurate geometry, and in which the material reflectivity/emissivity/transmissivity ratios of each surface can be controlled.

The first test was a 2-dimensional simulation based on the geometric layout shown in Figure 7. In this initial test we ignored the surroundings and only calculated the mean result based on the reflections from the parabolas in relation to a $2 \mathrm{D}$ point in space. A polar array of vectors from a point was created and values obtained from intersection with the hot or cold pipes or their reflections were calculated to obtain the mean temperature of all the vector intersections from the point. This 2D simulation technique was a first step in obtaining information for MRT distribution in the room, and its outcome was aimed to be gradient mesh illustrating this distribution. The arrangement of parabolas illustrated in Figure 7 was meant to optimize the view factor of cold surfaces towards one end of the room by directing all the cold parabolas towards it and hiding the view of the hot parabolas from that end as much as possible, while conversely optimizing the view factor of the hot surface towards the opposite end. In the middle of the room, neither view factor is optimized and 
a person standing there would be expected to receive a similar amount of hot and cold fluxes.

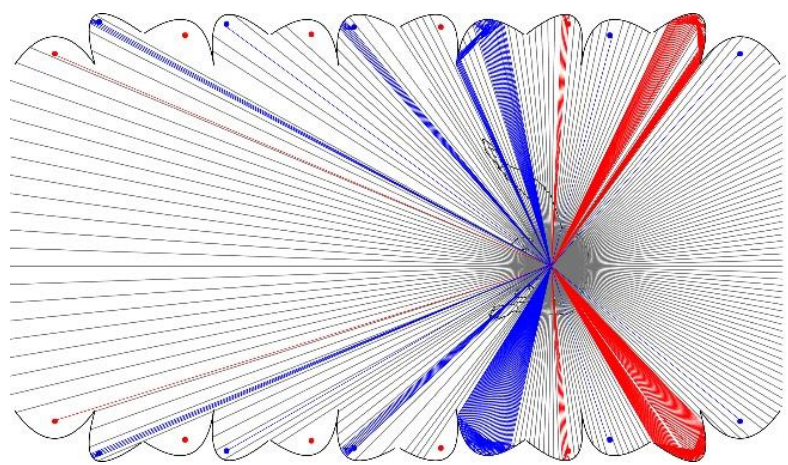

Figure 6: Diagram of heat flux reflected from cold and hot pipes onto a specific point in the room.

The next step was to construct a more robust and accurate 3D simulation, which included the full parameters of the actual physical experiment as built: the front and back walls and the wooden black-painted structural frame were assumed to have surface temperature equal to room temperature and high emissivity values.

The 3D-MRT simulation (Fig. 8) was produced in Grasshopper Algorithmic modeling software by using a $3 \mathrm{D}$ model of the space described above and assigning surface characteristics of temperature and emissivity to each modeled surface, represented digitally by mesh colors. The controlled parameters for the simulation were the surface temperature of the hot and cold pipes as well as the rest of the passive surfaces (taken from the measured data from the physical experiment described in the Results section). At each test point in the space, an array of vectors was created emanating from the point in the direction of all face-normals of a geodesic sphere concentric around that point. A vector array of 1250 vectors from each test point was then intersected with all the surfaces in the room (see Fig. 8). This method of using spherically arrayed vectoric representation is aimed to replace the view factor calculations that are based on solid angle projected in an analytical MRT solution (Hatefnia et al 2016). Based on a reflectivityemissivity-transmissivity ratio of the surrounding surfaces and their temperature value, the simulation registered a certain weighted value for that first intersection of each vector with a surface. Next, a reflection vector was drawn based on the angle of reflection from the surface and continued to bounce to the next surface. When intersecting a second another emissivity-weighted temperature was registered for the vector and so forth for the next intersections. After five bounces have been calculated to make any further intersections statistically negligible, the MRT of the point was calculated as the mean of all the registered temperature values weighted by their assigned emissivities. In this manner, the view factor of each surface, was statistically determined by the number of vectors hitting it and eliminated the need to calculate the view factor via solid angle.
The 3D simulation was constructed to either use a subdivided mesh surface with test points at the subdivision centroids to calculate its overall MRT of a complex surface such as the human body, or alternatively to look at the MRT value of a grid of test points in the entire room.

The numerical simulation results were validated against the black globe measurements taken in the physical experiment, at the two ends of the room and its center point at the median height of $1.2 \mathrm{~m}$.

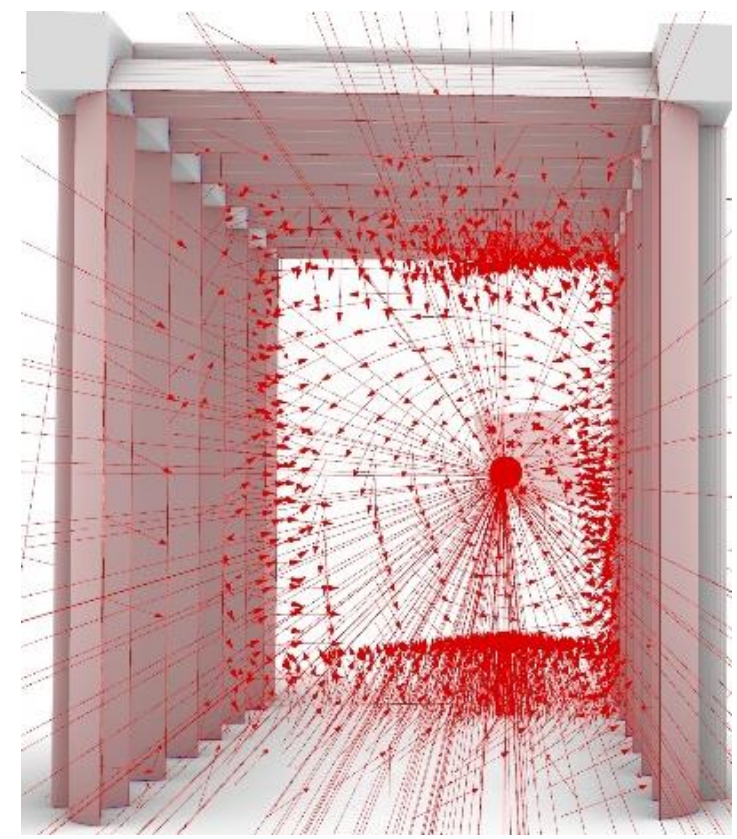

Figure 7:3D ray-tracing simulation: vectors emanate from a point and intersect with the surfaces of the room, and then bounce to a second intersection, a third and so forth.

\section{Results}

\section{Heat flux from a single pipe and a single parabola}

When maintaining a constant $\mathrm{d}$ distance, the deeper the parabola ( $a$ parameter increases), the higher percentage of rays from the surface of the pipe is captured by the parabolic surface and reflected in a parallel array (Fig. 9 ). The percentage of rays reflected by the parabola increases linearly but becomes asymptotic as the parabola becomes very steep and the ratio approaches 1 (full reflection). It was observed that because the heat flux leaving the pipe placed in the focal point became parallel as they are reflected by the parabolic surface (see Fig. 9), the parabolic reflection had the special characteristic that the radiance at a rectangular plane parallel to the parabola's face, rather than diminishing proportionally to the distance from the source according to the inverse square law ( $R \propto \frac{1}{d^{2}}$ ) where $R=$ radiance, measured at distance $d$ from source, is instead constant at any distance from it. 

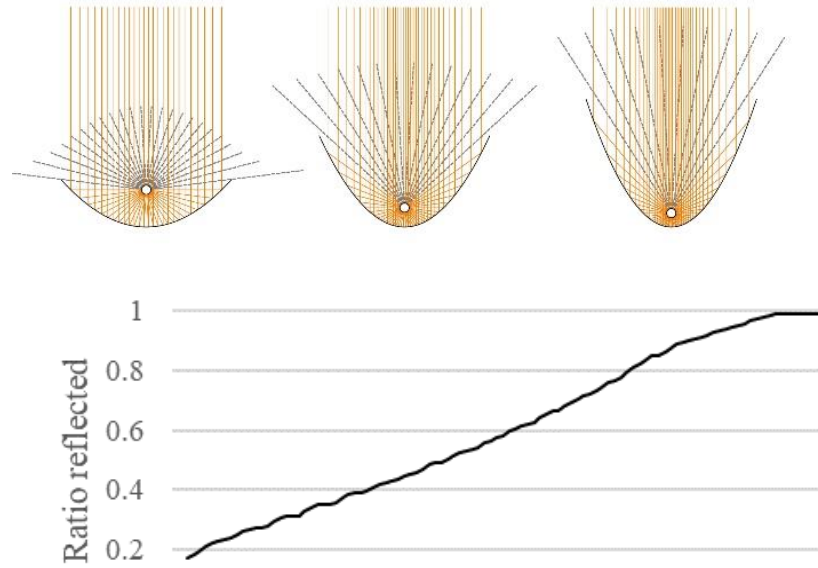

0

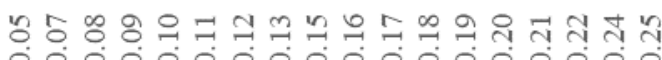

Slope of parabola $(a)$

Figure 8:diagram and plot of relation between parabolic shape and the ratio of heat flux reflected by the parabola.

This phenomenon is important for the analysis of the MRT simulation that follows, because essentially it means that by using a specific geometry constructed around a heat source, it is possible to magnify its effect on the total heat exchange in a room.

For the single parabola simulations of reflections from a point in the room, the illustrations in Figures 10 and 11 correspond to the illustrations shown in Figure 4 in the methods section: the orange lines show rays from a point in front of the parabola that hit the pipe after being reflected by the parabola; the grey lines show rays that miss the pipe when reflected back from the parabola surface. Therefore, as the point moves closer to the parabola (Figure 10), away from the point of convergence, the heat flux from the pipe to that point diminishes. This is shown in the graph below (note that irregularities in the mostly linear trend in the graph can be explained by the resolution of the simulation as well as by the fact that the pipe is taken as a region rather than a point).

Figure 11 illustrates the same procedure for a point moving sideways from the convergence point rather than closer to it. It is observed that moving sideways decreases the reflection at a much higher rate than moving closer.

The physical prototype shown for a cold pipe inside of a reflective aluminum parabola demonstrates the effect of moving sideways from the center of the pipe but also the challenge of achieving a full geometric fidelity with real surfaces (Fig. 12).

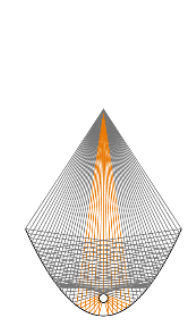

(a)

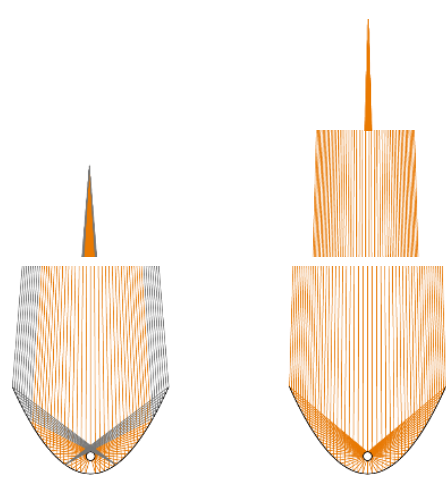

(b) (c)

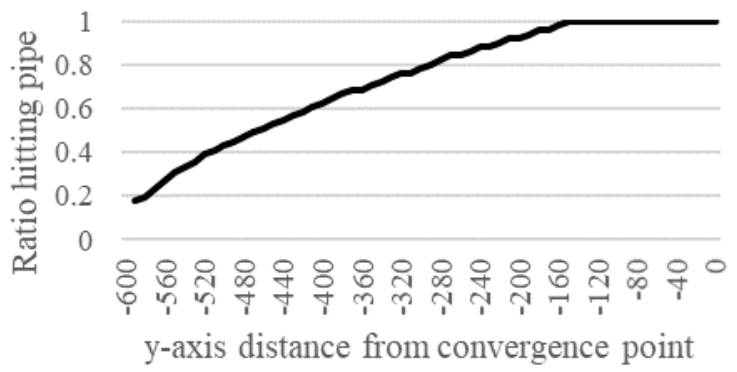

$(\mathrm{cm})$

Figure 9: diagram and plot of relation between position of a point on the $y$-axis the ratio of heat flux reflected by the parabola.

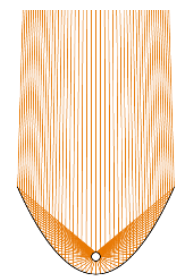

(c)

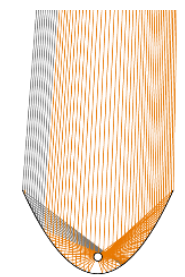

(d)

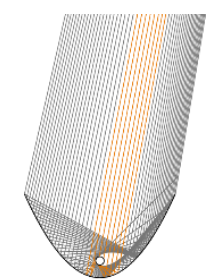

(e)

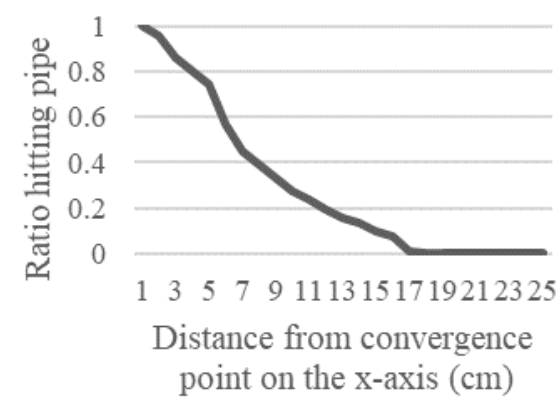

Figure 10: diagram and plot of relation between position of a point on the $x$-axis the ratio of heat flux reflected by the parabola. 


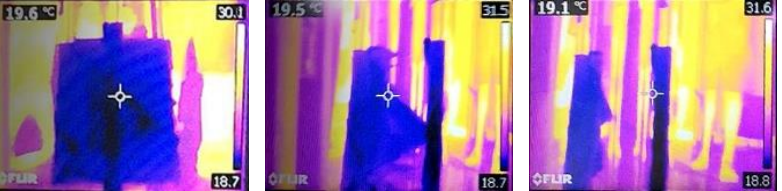

Figure 11: Infrared images of parabolic aluminium sheet with a cold pipe at the focal point. Camera moving sideways showing reflection effect diminishing.

\section{Room-Scale Physical Prototype}

The physical prototype was constructed as part of the 2017 Seoul Biennale of Architecture and Urbanism in Korea (cite event publication/book). It was constructed and tested initially in August and September, and after the exhibition was closed more measurements with globe thermometers and more sensors were carried out in January 2018.

The measurements in the room were as follows:

1. System Surface temperatures were measured by FLIR thermal camera and were controlled to be 11.0 and $39.0^{\circ} \mathrm{C}$.

2. Flowrate in the system was measured by observing volume displacement over time with constant speed pumps at $0.9 \mathrm{~L} / \mathrm{s}$ and $0.16 \mathrm{~L} / \mathrm{s}$ for the heat pumps.

3. Tank temperatures were measured to be $9.5^{\circ} \mathrm{C}$ and $48{ }^{\circ} \mathrm{C}$, and the supply and return temperatures at 10.2 and $10.4{ }^{\circ} \mathrm{C}$ for the cold side and 39.1 and $39.7{ }^{\circ} \mathrm{C}$ on the hot side.

4. Temperature and humidity was measured in the room during the winter experiments to be $21^{\circ} \mathrm{C}$ and $32 \% \mathrm{RH}$.

5. Black globes at the front, middle and back of the setup measured, $18.8^{\circ} \mathrm{C}, 20.7^{\circ} \mathrm{C}$, and $21.9^{\circ} \mathrm{C}$ while the air temperature was measured to be 19.0, 22.2 and $22.7^{\circ} \mathrm{C}$ across the space

There was not enough time to run a controlled thermal comfort study, but occupants did note the change in temperature during the exhibition, though it was small. A change in MRT of 3 degrees in that environment would be equivalent to perceiving a change in temperature of about $1.5 \mathrm{deg}$ C.

\section{MRT Simulation Results}

The 2D simulation result in Fig. 13 shows a 2D mesh with color gradient based on the view factor per point from hot and cold rays reflected to the point from the cold and hot pipes. As explained in the method section, this was an initial simulation which did not include all the surfaces in the room and only calculated the reflective parabolas in $2 \mathrm{D}$. The results are thus completely symmetrical around the $\mathrm{x}$-axis and symmetrically inverted around the y-axis. Note the distribution pattern, where the one end of the room is coldest and the other is hottest due to the direction at which the parabolas were pointing (see Fig. 7). Figure 14 shows the results for the 3D MRT simulation, with gradient meshes of temperature distribution between $18^{\circ} \mathrm{C}$ to $24^{\circ} \mathrm{C}$.

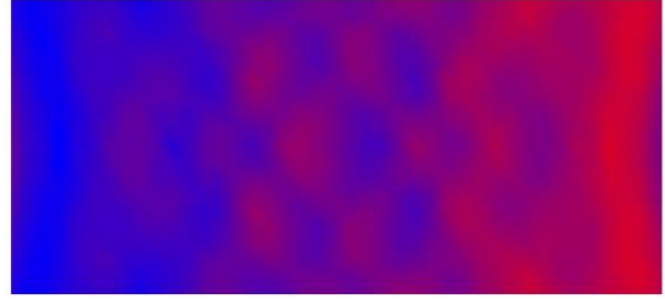

Figure 12: 2D mesh.

It was observed that the most extreme temperature difference occurs at the upper portion of the room, close to the reflective parabolas and pipes at the ceiling and furthest from the floor, which is assumed to be room temperature in the simulation. Conversely, the gradient mesh with test points closest to the floor, produced the most uniform temperature distribution with only 3 degrees difference between the hot side and the cold side. Points a,b,c shown in Figure 14 are comparable to the measurements taken with the black globe thermometers at the same locations. The simulation shows a Mean Radiant Temperature difference of 4 degrees Celsius between point a and point $\mathrm{c}$ while point $\mathrm{b}$ is exactly midway between them. The black globe reading shows a difference of 3 degrees Celsius which is within the precision of the black globe thermometer.

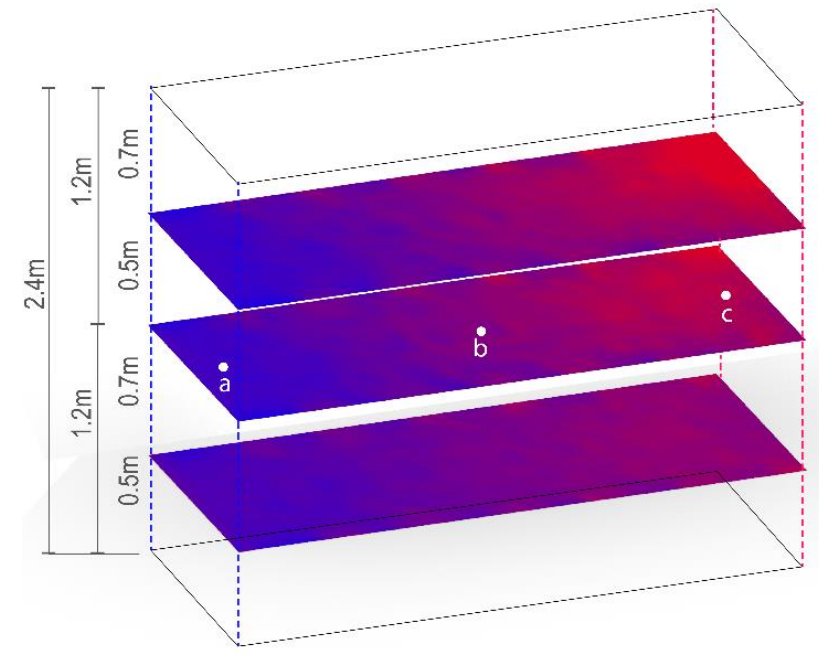

Figure 13:Gradient Mesh results based on MRT values at multiple points at three different heights in the room.

The results show a limited range of mean radiant temperature in the room: $3^{\circ} \mathrm{C}$ in the physical prototype according to the black globe measurements and maximum of $6^{\circ} \mathrm{C}$ difference between the coldest and hottest spots in the room per the digital the simulation. This limited range is due to the confines of the experimental setup, specifically the bidirectional emission in the attempt to heat and cool different parts of the same space simultaneously. Because of this, the occupant's body was always exposed to both hot and cold surfaces at the same time, and the MRT variations were dependent on a restricted range view factor ratios. For instance, the view factor of all hot surfaces combined even at the highest MRT point in the room was still less than half of the total sphere. 
Another practical limitation of the setup was during construction, when small imperfections in the material surface and slight misalignments from the parabolic trough guides resulted in imperfect reflection. Additionally, highly reflective materials for thermal radiation (reflectivity $>0.9$ ) are often expensive. However, ray tracing models illustrate a complementary finding, whereby deeper parabolas naturally reflect more radiation due to natural material properties, namely emissivity decreasing as the angle of incidence becomes shallower. The angle of incidence between radiation emitted from the focal pipe is often at shallow angles in relation to the parabolic reflector, which helps increase the spectral reflectivity, beyond the hemispherical emissivity calculated for orthogonal radiation. This benefit is for steep parabolas only.

\section{Conclusion}

It was successfully demonstrated that it is possible to intentionally create a non-uniform MRT distribution in an indoor environment by manipulating the geometry and material properties of surfaces in the room in relation to heat sources. Moreover, by characterizing the properties of diffuse radiation from a heat source, it is possible to design its surrounding in a form that maximizes its perceived radiant effect on a human occupant.The experimental setup with bidirectional emission in the attempt to heat and cool different parts of the same room at the same time reduced the optimal view factor that was achievable, and therefore produced limited MRT variations. In a real-world scenario however, one would probably wish to maximize either the cooling or the heating effect on a person and not both simultaneously. Thus, it would be feasible to make a significant impact on the thermal comfort of a person in one segment of a room using radiation heating or cooling, while allowing the rest of the room to remain in relatively unaffected ambient temperature. Providing more than one indoor climate within the same space poses significant energy savings in comparison to airbased systems which must account for air diffusion throughout a room. Furthermore, since the methods we have demonstrated allow one to intentionally direct radiant fluxes towards a person's location, if the surrounding surfaces and heat sources locations were designed in conjunction with the room's functional uses and furniture layout, they could enhance the local thermal comfort of the occupants.

Future advancements of this study will include a 3D MRT simulation which will sum the total heat gain by a human body in space using a discretized body mesh as the source for the diffuse radiation rather than a geodesic sphere. Additionally, characterization of the relationship between the heat pump efficiency and the produced MRT results is necessary.

\section{Acknowledgements}

We would like to thank Yshai Yudekovitz for his important contribution to the Grasshopper 3D code.

\section{References}

Boetcher, S. K. (2014). Natural convection from circular cylinders. Springer International Publishing.

Cohen, M. F., \& Greenberg, D. P. (1985, July). The hemi-cube: A radiosity solution for complex environments. In ACM Siggraph Computer Graphics (Vol. 19, No. 3, pp. 31-40). ACM.

Guo, H., Teitelbaum, E., Houchois, N., Bozlar, M., \& Meggers, F. (2018). Revisiting the use of globe thermometers to estimate radiant temperature in studies of heating and ventilation. Energy and Buildings, 180, 83-94.

Hatefnia, N., A. Barakati, M. Ghobad, and A.E. Panah. (2016). "A Novel Methodology to Assess Mean Radiant Temperature in Complex Outdoor Spaces." In PLEA 2016 Los Angeles. 32th International Conference on Passive and Low Energy Architecture.

Heck, C. M. (1941). U.S. Patent No. 2,234,122. Washington, DC: U.S. Patent and Trademark Office.

Huizenga, C., Hui, Z., \& Arens, E. (2001). "A model of human physiology and comfort for assessing complex thermal environments." Building and Environment, 36(6), 691-699.

Li, J., \& Tarasuk, J. D. (1992). Local free convection around inclined cylinders in air: an interferometric study. Experimental thermal and fluid science, 5(2), 235-242.

Matzarakis, A., Rutz, F., \& Mayer, H. (2007). Modelling radiation fluxes in simple and complex environments-application of the RayMan model. International journal of biometeorology, 51(4), 323-334.

McLauchlan, D.J., and B. Dutt (2008): "BACnet [R] for green library." ASHRAE Journal 50, no. 11 B37-B37.

Meggers, F., Guo, H., Teitelbaum, E., Aschwanden, G., Read, J., Houchois, N., ... \& Calabrò, E. (2017). The Thermoheliodome- "Air conditioning" without conditioning the air, using radiant cooling and indirect evaporation. Energy and Buildings, 157, 1119.

Roulet, C. A., Foradini, F., \& Westphal, E. (2005). RADIACONF: A design tool for locating and sizing radiant panels to improve comfort (No. CONF, pp. 409-414).

Schiavon, S., Hoyt, T., \& Piccioli, A. (2014, August). Web application for thermal comfort visualization and calculation according to ASHRAE Standard 55. In Building Simulation (Vol. 7, No. 4, pp. 321-334). Tsinghua University Press. 